\title{
Excitation wavelength-dependent nearly pure white light-emitting crystals from a single gold(I)-containing complex
}

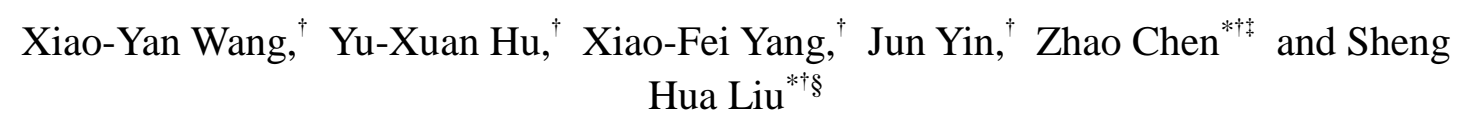

${ }^{\dagger}$ Key Laboratory of Pesticide and Chemical Biology, Ministry of Education, College of Chemistry, Central China Normal University, Wuhan 430079, PR China. E-mail: chshliu@mail.ccnu.edu.cn; Fax:+86-27-67867725; Tel: +86-27-67867725.

" Jiangxi Key Laboratory of Organic Chemistry, Jiangxi Science and Technology Normal University, Nanchang 330013,PR China.E-mail: chenzhao666@126.com; Fax: +86-791-83831996; Tel: +86-791-83831996.

$\S$ State Key Laboratory of Structural Chemistry, Fujian Institute of Research on the Structure of Matter, Chinese Academy of Sciences, Fuzhou 350002, P. R. China. 


\section{Contents}

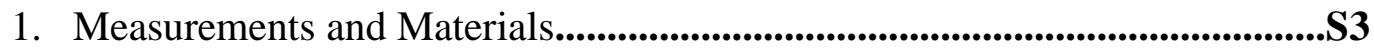

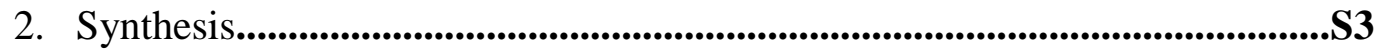

3. Copies of NMR spectra.............................................................................................S4

4. Figures S8-S13..................................................................................................................S7

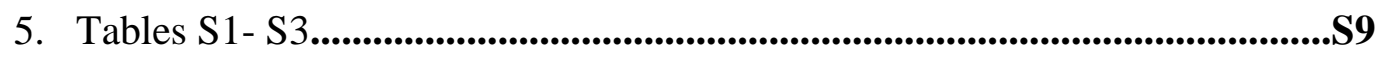




\section{Measurements and Materials}

NMR spectra were collected on American Varian Mercury Plus 400 spectrometer (400 MHz) or Varian Mercury Plus $600 \mathrm{MHz}$ instrument. ${ }^{1} \mathrm{H}$ and ${ }^{13} \mathrm{C}$ NMR chemical shifts were relative to TMS. ${ }^{19} \mathrm{~F}$ NMR chemical shifts were relative to $\mathrm{C}_{6} \mathrm{~F}_{6}(\delta=$ -163.00). Mass spectra were recorded on a Bruker ultrafleXtreme MALDI-TOF-TOF mass spectrometer and 6224 TOF LC/MS spectrometer. PL spectra were obtained by Edinburgh FLS1000 spectrometer with Xe lamp as excitation light source. Decay curves of crystals were measured by Edinburgh FLS1000 spectrometer with $\mu$ F2 lamp as excitation light source. Photos of crystals were recorded by CYTATION 5 (BioTek) CIE chromaticity coordinates were calculated by CIE 1931. The X-ray crystal-structure data were obtained by the Bruker APEX DUO CCD system. All manipulations were carried out in argon atmosphere by using standard Schlenk techniques, unless otherwise stated. All starting meterials and reagents were purchased from Shen Shi Hua Gong Company (China) and used without further purification. The reaction requiring heating or reflux is carried out in an oil bath.

\section{Synthesis}

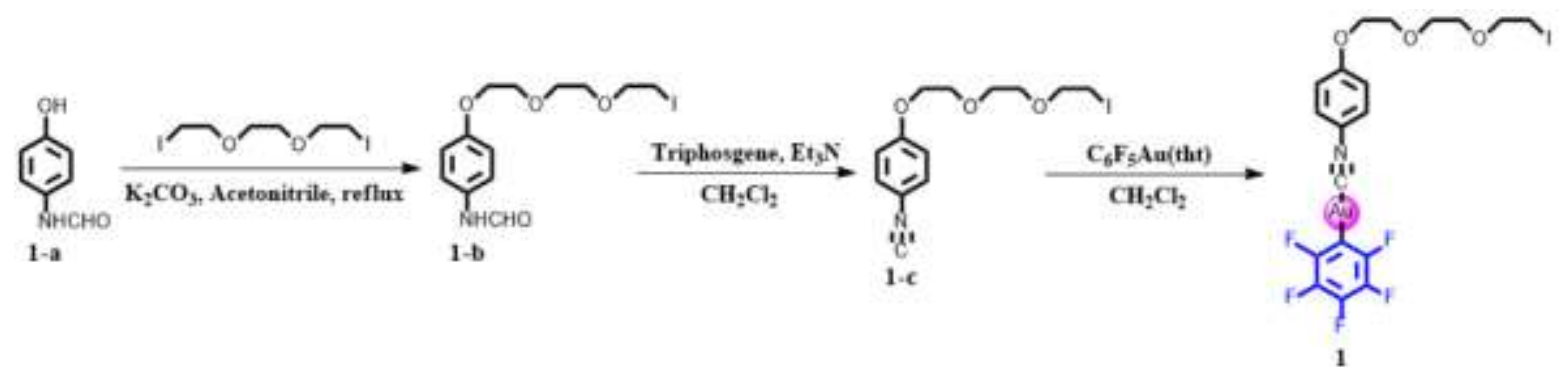

Scheme S1 Synthetic route to the gold(I) complex 1 (tht = tetrahydrothiophene).

Synthesis of 1-b: N-(4-hydroxyphenyl)formamide (1-a) $(617.80 \mathrm{mg}, 4.5 \mathrm{mmol})$ and potassium carbonate $(1.86 \mathrm{~g}, 13.5 \mathrm{mmol})$ were dissolved in $50 \mathrm{~mL}$ acetonitrile and stirred for several minutes. Then 1,2-bis(2-iodoethoxy)ethane (5.00 g, $13.5 \mathrm{mmol})$ were added. The mixture was refluxed for $36 \mathrm{~h}$. After completion of present reaction, the solvent was removed by vacuum distillation. The residual mixture was extracted with ethyl acetate $(3 \times 50 \mathrm{~mL})$. The combined organic layers were washed with brine, dried $\left(\mathrm{Na}_{2} \mathrm{SO}_{4}\right)$, and concentrated in vacuo. The residues were purified by column chromatography, affording the expected oil yellow liquid 1-b $(1175.52 \mathrm{mg})$ in a yield of $68.8 \% .{ }^{1} \mathrm{H}$ NMR (400 MHz, Chloroform- $d$ ) $\delta$ 8.51-8.30 (m, 1H), $7.44(\mathrm{~d}, J=8 \mathrm{~Hz}$, 
1H), 7.19-7.01 (m, 2H), $6.91(\mathrm{t}, J=10 \mathrm{~Hz}, 2 \mathrm{H}), 4.13(\mathrm{~s}, 2 \mathrm{H}), 3.90-3.85(\mathrm{~m}, 2 \mathrm{H})$, 3.78-3.69 (m, 6H), $3.26(\mathrm{t}, J=8 \mathrm{~Hz}, 2 \mathrm{H}) .{ }^{13} \mathrm{C}$ NMR (101 MHz, Chloroform- $\left.d\right) \delta$ $163.0,159.3,156.2,155.2,130.3,121.4,114.5,71.6,70.4,69.8,69.4,67.3,2.9$. HRMS (LC-MS) $(m / z):[\mathrm{M}+\mathrm{Na}]^{+}$, calcd. for $\mathrm{C}_{13} \mathrm{H}_{18} \mathrm{INO}_{4}, 402.0173$; found: 402.0183 .

Synthesis of 1-c: $\mathrm{A} \mathrm{CH}_{2} \mathrm{Cl}_{2}$ suspension $(15 \mathrm{ml})$ of $\mathbf{1 - b}(758.06 \mathrm{mg}, 2.0 \mathrm{mmol})$ and triethylamine $(5 \mathrm{ml})$ was cooled to $0^{\circ} \mathrm{C}$. To the mixture was added dropwise a $\mathrm{CH}_{2} \mathrm{Cl}_{2}$ solution $(10 \mathrm{ml})$ of triphosgene $(652.85 \mathrm{mg}, 2.2 \mathrm{mmol})$. The mixture was refluxed for $3 \mathrm{~h}$, then $10 \%$ aq. $\mathrm{Na}_{2} \mathrm{CO}_{3}(50 \mathrm{ml})$ was added dropwise at room temperature. The mixture was extracted with dichloromethane $(3 \times 20 \mathrm{~mL})$. The combined organic layers were washed with brine, dried $\left(\mathrm{Na}_{2} \mathrm{SO}_{4}\right)$, and concentrated in vacuo. The residues were purified by column chromatography, affording the expected product 1-c $509.04 \mathrm{mg}$ in a yield of 70.5\%. ${ }^{1} \mathrm{H}$ NMR $(600 \mathrm{MHz}$, Chloroform- $d) \delta 7.30(\mathrm{~d}, J=4$ $\mathrm{Hz}, 2 \mathrm{H}), 6.90(\mathrm{~d}, J=4 \mathrm{~Hz}, 2 \mathrm{H}), 4.14(\mathrm{~s}, 2 \mathrm{H}), 3.88(\mathrm{~s}, 2 \mathrm{H}), 3.77-3.68(\mathrm{~m}, 6 \mathrm{H})$, 3.27-3.25 (m, 2H). ${ }^{13} \mathrm{C}$ NMR (101 MHz, Chloroform- $d$ ) $\delta 162.5,159.0,127.7,115.1$, 71.9, 70.8, 70.2, 69.5, 67.7, 2.9. HRMS (LC-MS) $(\mathrm{m} / \mathrm{z}):[\mathrm{M}+\mathrm{Na}]^{+}$, calcd. for $\mathrm{C}_{13} \mathrm{H}_{16} \mathrm{INO}_{3}, 384.0067$; found: 384.0074 .

Synthesis of 1: A mixture of $\mathrm{C}_{6} \mathrm{~F}_{5} \mathrm{Au}($ tht $)(237.40 \mathrm{mg}, 0.525 \mathrm{mmol})$ and 1-c (180.59 mg, $0.5 \mathrm{mmol}$ ) was stirred in $\mathrm{CH}_{2} \mathrm{Cl}_{2}(20 \mathrm{~mL})$ overnight at room temperature. After completion of present reaction, the solvent was evaporated. A small amount of $\mathrm{CH}_{2} \mathrm{Cl}_{2}$ was added, and then a lot of $n$-hexane was added. Collecting the white solid product by suction filtration. Yield $=64 \%(232.09 \mathrm{mg}) .{ }^{1} \mathrm{H}$ NMR $(400 \mathrm{MHz}$, Chloroform- $d$ ) $\delta 7.48(\mathrm{~d}, J=12 \mathrm{~Hz}, 2 \mathrm{H}), 7.01(\mathrm{~d}, J=8 \mathrm{~Hz}, 2 \mathrm{H}), 4.20(\mathrm{t}, J=4 \mathrm{~Hz}, 2 \mathrm{H})$, $3.91(\mathrm{t}, J=4 \mathrm{~Hz}, 2 \mathrm{H}), 3.78-3.69(\mathrm{~m}, 6 \mathrm{H}), 3.27(\mathrm{t}, J=6 \mathrm{~Hz}, 2 \mathrm{H}) .{ }^{19} \mathrm{~F} \mathrm{NMR}$ $\left(\right.$ Chloroform- $d$ ) $\delta-115.97,-157.59,-162.43 .{ }^{13} \mathrm{C}$ NMR (101 MHz, Chloroform- $d$ ) $\delta$ $161.0,156.8,150.8,150.5,148.5,148.3,140.6,138.1,135.9,128.4,116.8,115.9$, 72.0, 70.9, 70.3, 69.5, 68.1, 2.8. HRMS (Maldi-TOF-MS) $(\mathrm{m} / \mathrm{z}):[\mathrm{M}+\mathrm{Na}]^{+}$, calcd. for $\mathrm{C}_{19} \mathrm{H}_{16} \mathrm{AuF}_{5} \mathrm{INO}_{3}, 747.9653$; found, 747.9651.

\section{Copies of NMR spectra}




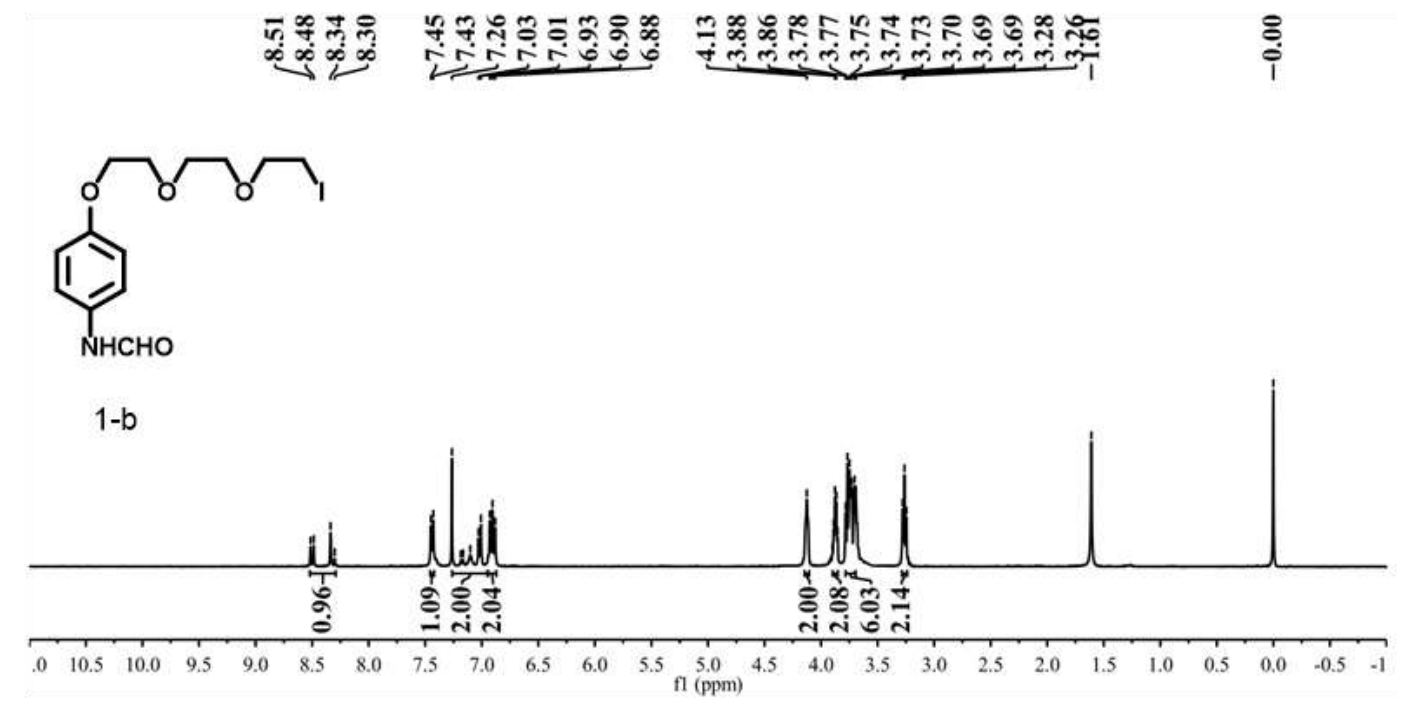

Figure S1. ${ }^{1} \mathrm{H}$ NMR spectrum of $\mathbf{1 - b}$ in $\mathrm{CDCl}_{3}$ at room temperature.

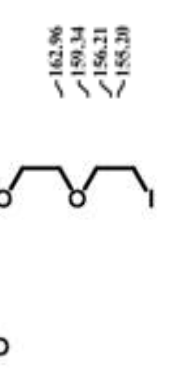

$1-b$
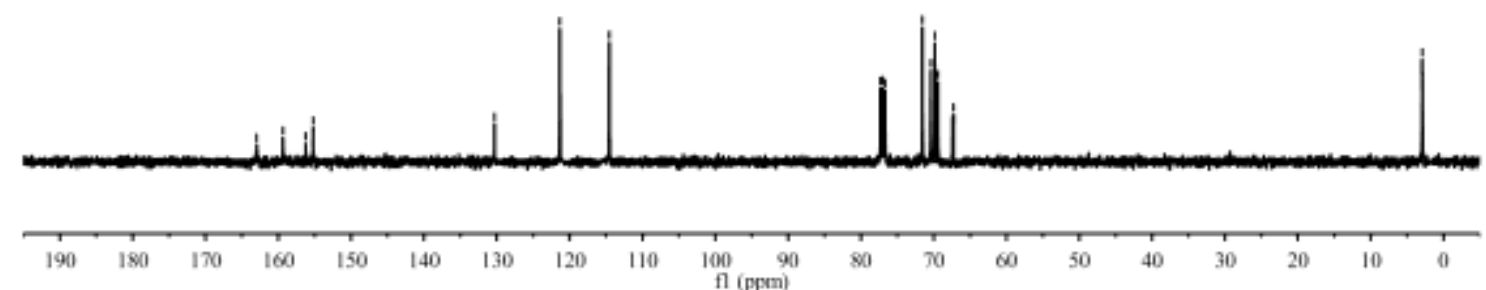

Figure S2. ${ }^{13} \mathrm{C}$ NMR spectrum of 1-b in $\mathrm{CDCl}_{3}$ at room temperature.

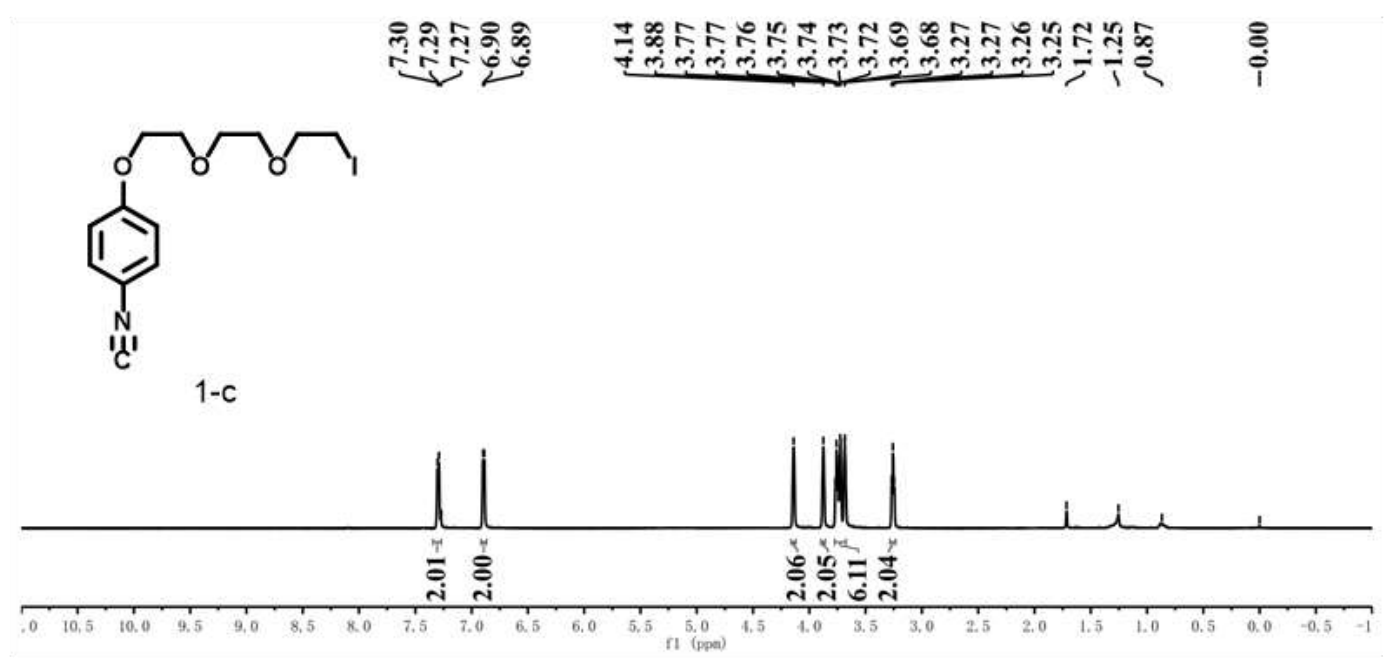

Figure S3. ${ }^{1} \mathrm{H}$ NMR spectrum of 1-c in $\mathrm{CDCl}_{3}$ at room temperature. 


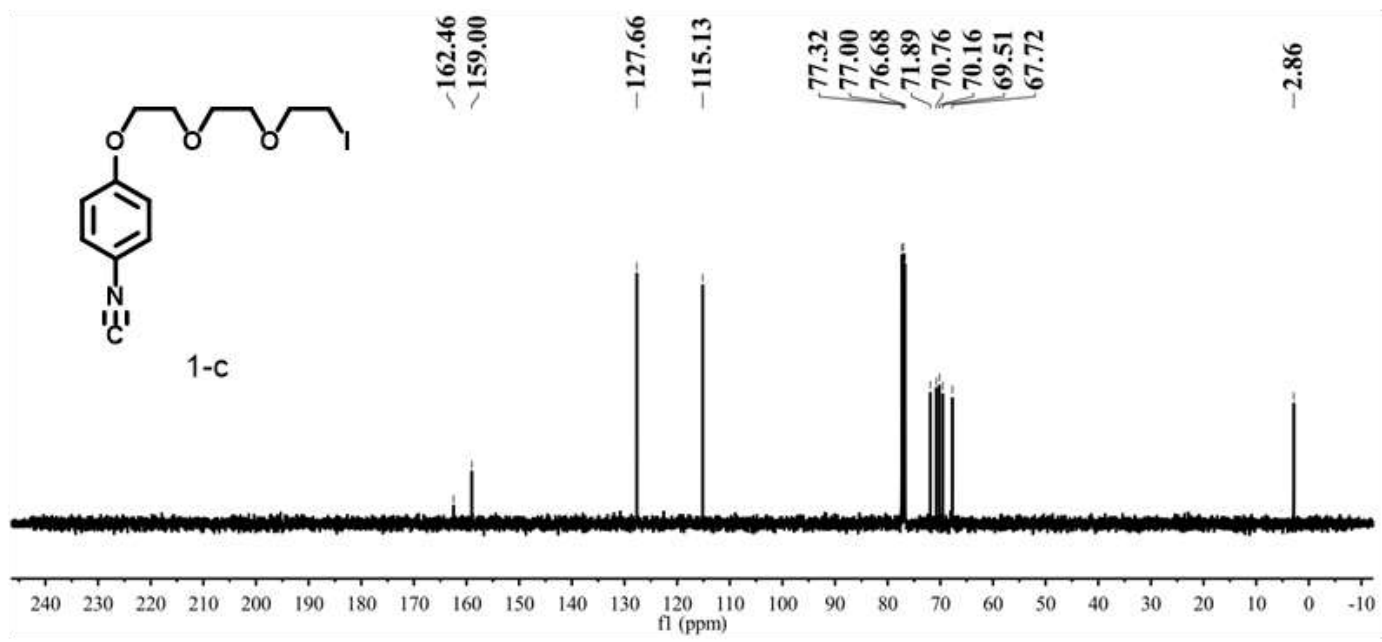

Figure S4. ${ }^{13} \mathrm{C}$ NMR spectrum of 1-c in $\mathrm{CDCl}_{3}$ at room temperature.

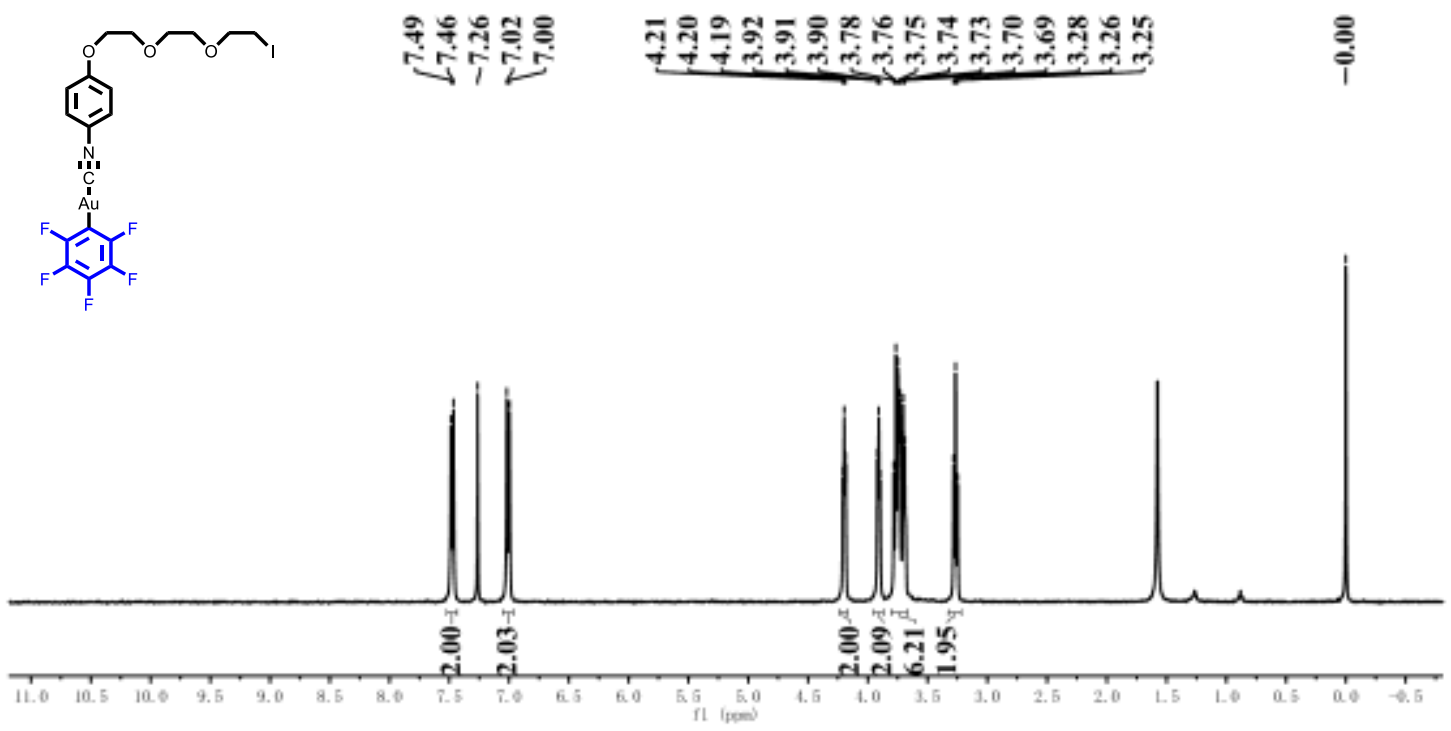

Figure S5. ${ }^{1} \mathrm{H}$ NMR spectrum of $\mathbf{1}$ in $\mathrm{CDCl}_{3}$ at room temperature.
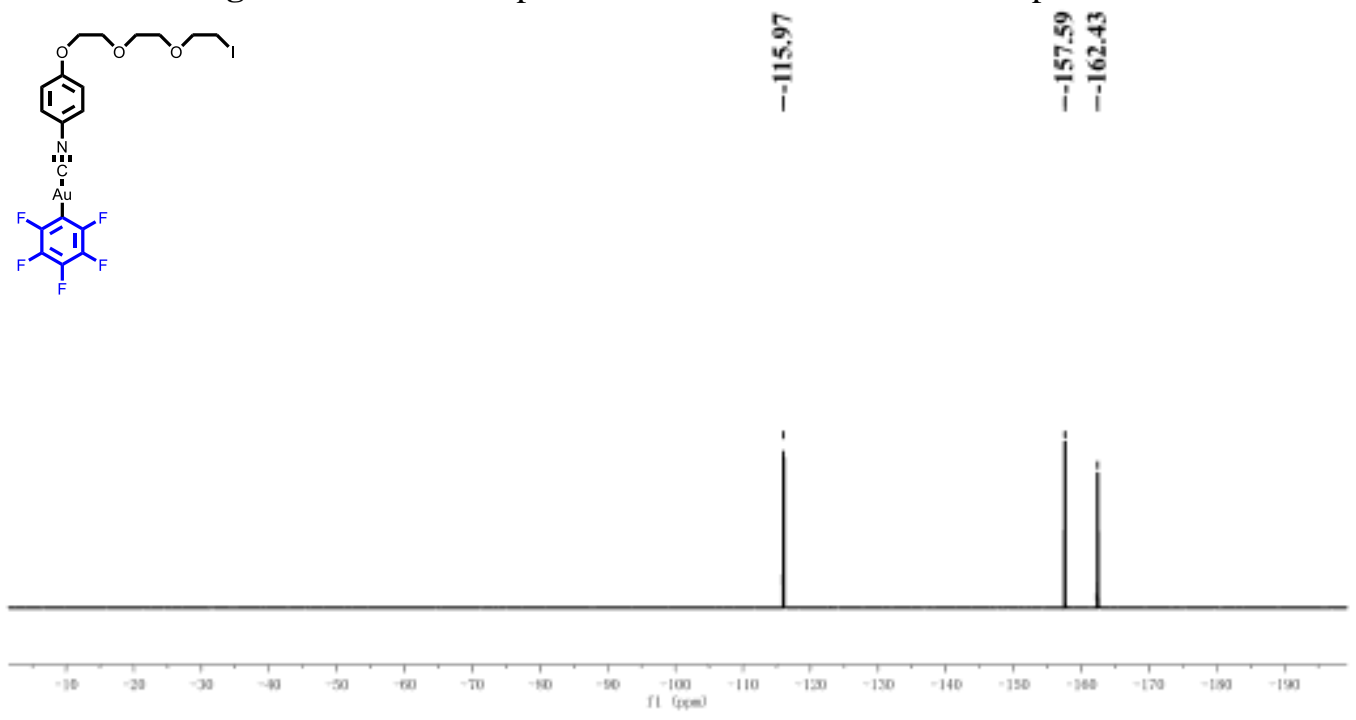

Figure S6. ${ }^{19} \mathrm{~F}$ NMR spectrum of $\mathbf{1}$ in $\mathrm{CDCl}_{3}$ at room temperature. 


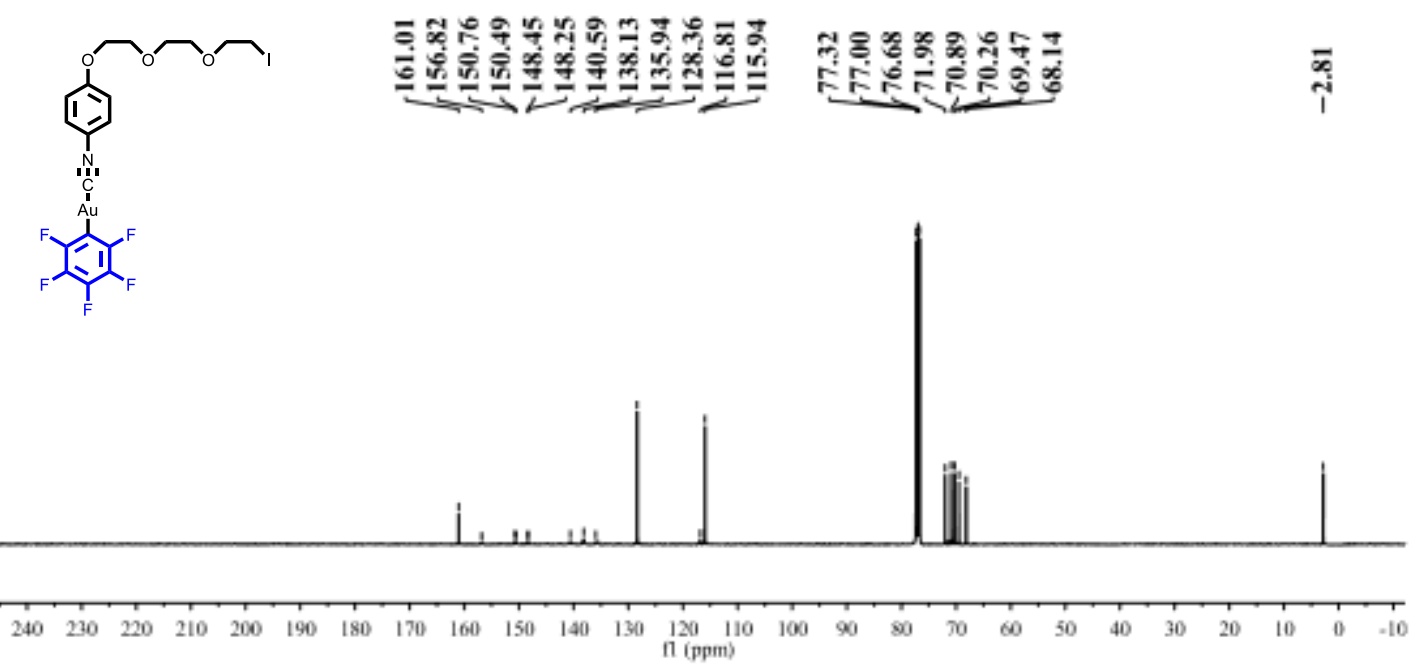

Figure S7. ${ }^{13} \mathrm{C}$ NMR spectrum of 1 in $\mathrm{CDCl}_{3}$ at room temperature.

\section{Figures S8-S13}
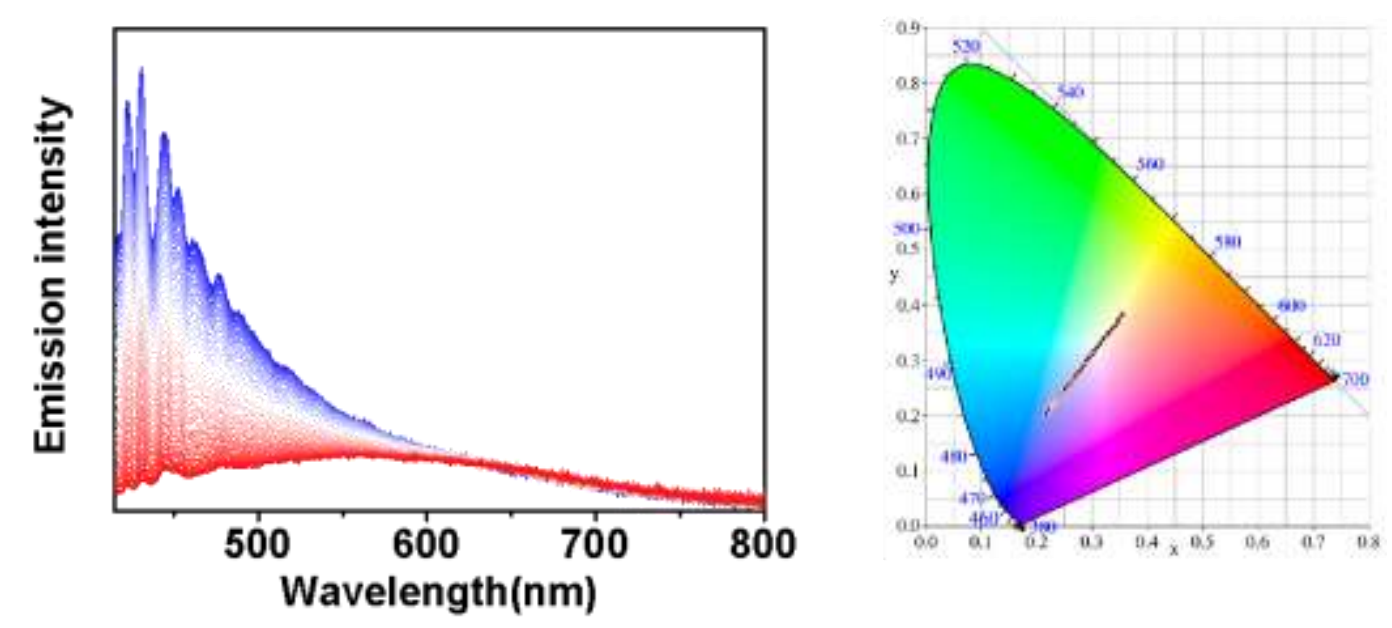

Figure S8. (a) PL spectra of the crystals under different excitation wavelengths (excitation wavelength range: 400 (blue line)-405 (red line) nm) at $77 \mathrm{~K}$ with a wavelength interval of $0.1 \mathrm{~nm}$; (b) CIE chromaticity coordinates in CIE-1931 chromaticity diagram of crystals samples at $77 \mathrm{~K}$ were excited over the wavelength range from 400 to $405 \mathrm{~nm}$ with a wavelength interval of $0.1 \mathrm{~nm}$. 


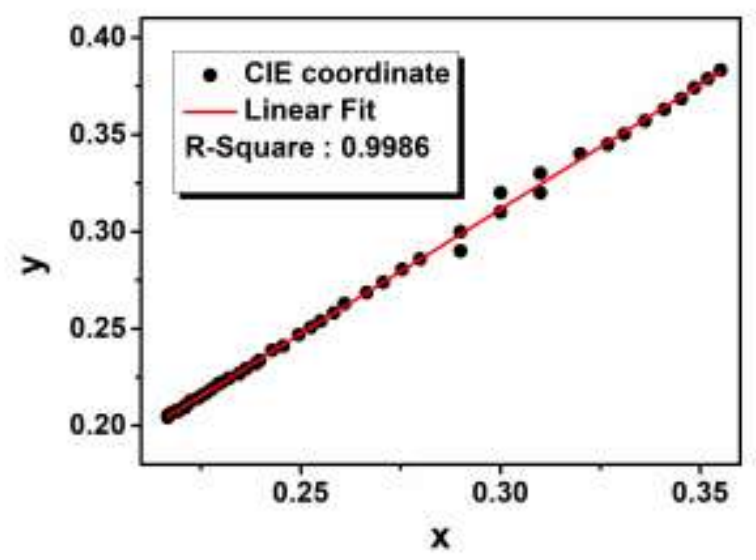

Figure S9. The linear relationship of the corresponding CIE chromaticity coordinates $x$ and $y$ at $77 \mathrm{~K}$.

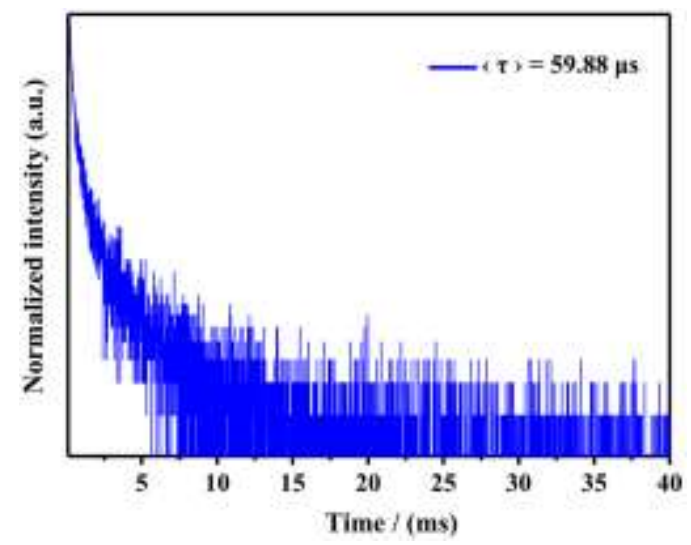

Figure S10. Decay curve of the crystals at the peak emission wavelength of $580 \mathrm{~nm}$ at $77 \mathrm{~K}$. Excitation wavelength $=404.1 \mathrm{~nm}$.

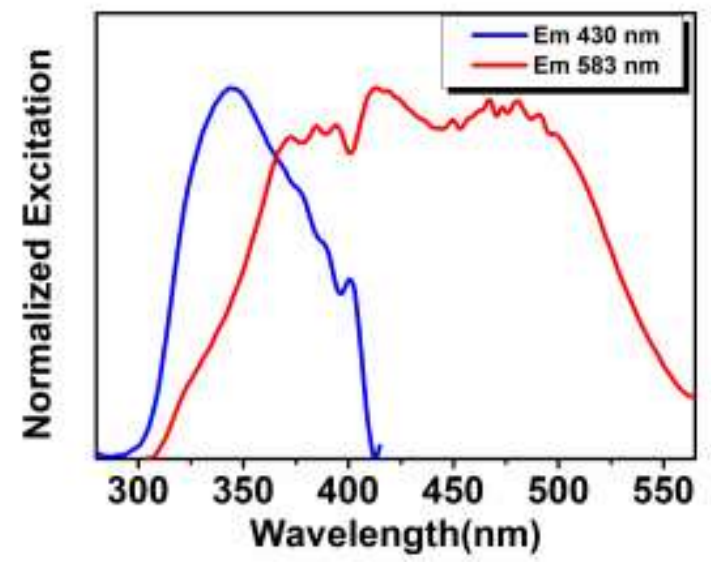

Figure S11. Excitation spectra of complex 1 at different emission wavelengths at room temperature. 


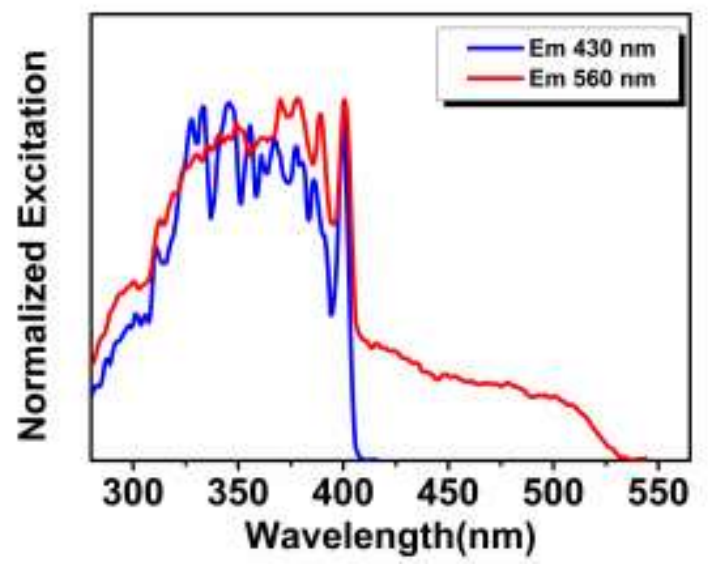

Figure S12. Excitation spectra of complex 1 at different emission wavelengths at $77 \mathrm{~K}$.
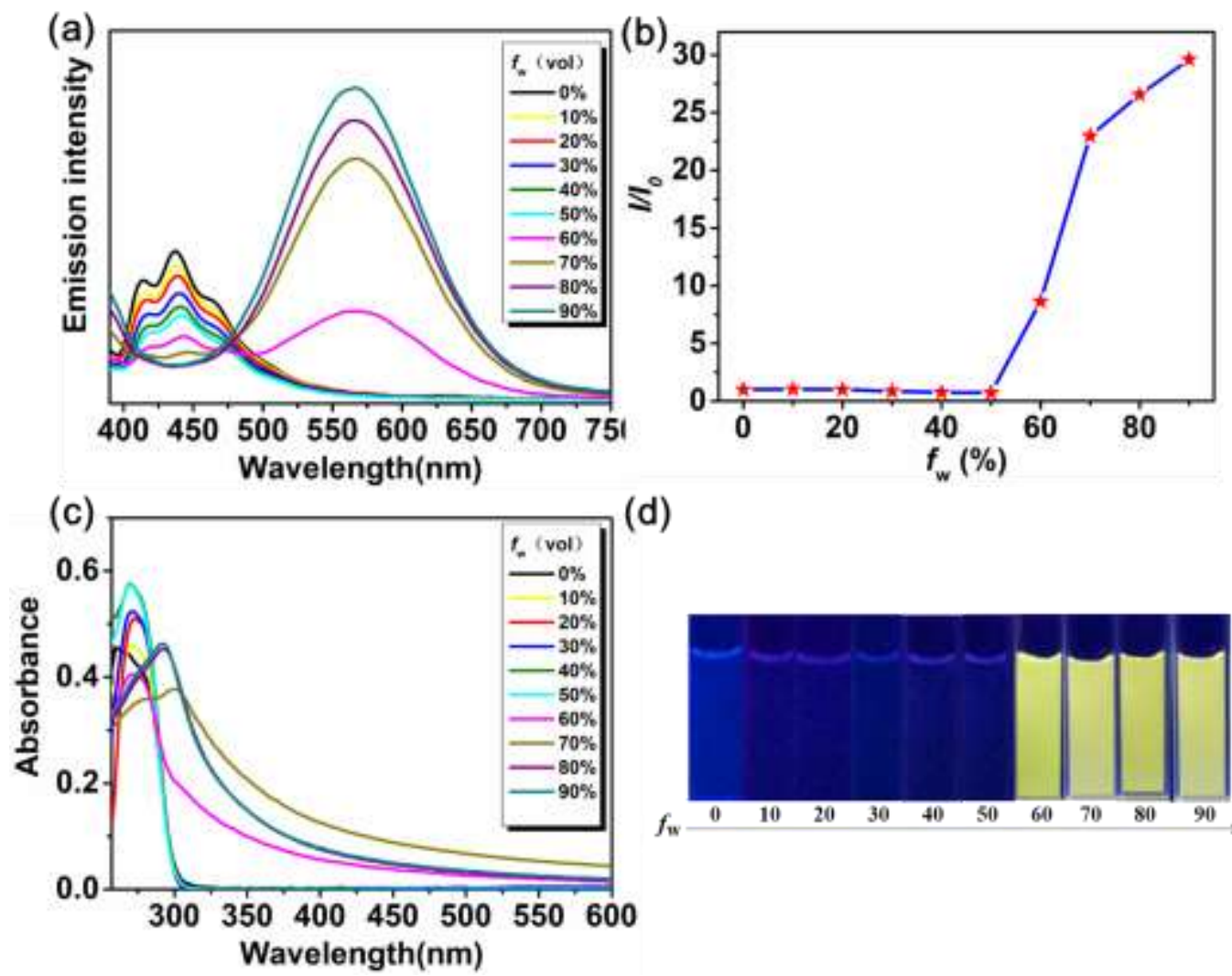

(d)

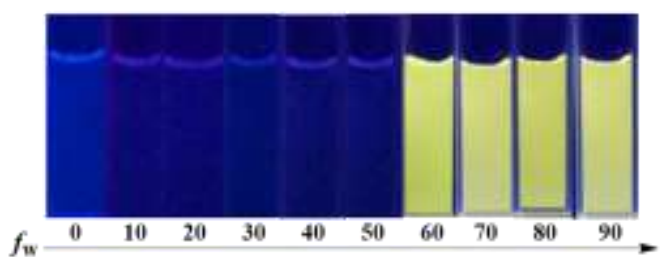

Figure S13. PL spectra (a) and UV-Vis absorption spectra (c) of the complex $1(20 \mu \mathrm{M})$ in DMF-water mixtures with different volume fractions of water (0-90\%), excitation wavelength: $365 \mathrm{~nm}$. (b) Changes in the emission intensity of complex 1 at $568 \mathrm{~nm}$ in DMF-water mixtures with different volume fractions of water (0-90\%). (d) The fluorescence images of $\mathbf{1}$ in DMF-water mixtures with different water content under $365 \mathrm{~nm}$ UV light.

\section{Tables S1- S3}

Table S1 Structure determination summary for the complex 1. 


\begin{tabular}{|c|c|}
\hline & mo_190701d_0m \\
\hline \multicolumn{2}{|r|}{ Crystal data } \\
\hline Chemical formula & $\mathrm{C}_{19} \mathrm{H}_{16} \mathrm{AuF}_{5} \mathrm{INO}_{3}$ \\
\hline$M_{\mathrm{r}}$ & 725.19 \\
\hline $\begin{array}{c}\text { Crystal system, space } \\
\text { group }\end{array}$ & Monoclinic, $P 2_{1} / n$ \\
\hline Temperature $(\mathrm{K})$ & 296 \\
\hline$a, b, c(\AA)$ & $14.392(2), 7.4267(11), 20.043(3)$ \\
\hline$\beta\left(^{\circ}\right)$ & $96.508(2)$ \\
\hline$V\left(\AA^{3}\right)$ & $2128.6(5)$ \\
\hline$Z$ & 4 \\
\hline Radiation type & Mo $K \alpha$ \\
\hline$\mu\left(\mathrm{mm}^{-1}\right)$ & 8.43 \\
\hline Crystal size (mm) & $0.12 \times 0.1 \times 0.1$ \\
\hline \multicolumn{2}{|r|}{ Data collection } \\
\hline Diffractometer & Bruker $A P E X$-II CCD \\
\hline Absorption correction & $\begin{array}{l}\text { Multi-scan } \\
\text { SADABS2008/1 (Bruker,2008) was used for absorption correction. } \\
\text { wR2(int) was } 0.1483 \text { before and } 0.0728 \text { after correction. The Ratio of } \\
\text { minimum to maximum transmission is } 0.3287 \text {. The } \lambda / 2 \text { correction factor } \\
\text { is } 0.0015 \text {. }\end{array}$ \\
\hline$T_{\min }, T_{\max }$ & $0.245,0.746$ \\
\hline $\begin{array}{l}\text { No. of measured, } \\
\text { independent and } \\
\text { observed }[I>2 \sigma(I)] \\
\text { reflections }\end{array}$ & $13912,3934,3541$ \\
\hline$R_{\text {int }}$ & 0.048 \\
\hline$(\sin \theta / \lambda)_{\max }\left(\AA^{-1}\right)$ & 0.606 \\
\hline \multicolumn{2}{|r|}{ Refinement } \\
\hline $\begin{array}{c}R\left[F^{2}>2 \sigma\left(F^{2}\right)\right], \\
w R\left(F^{2}\right), S\end{array}$ & $0.046, \quad 0.126, \quad 1.12$ \\
\hline No. of reflections & 3934 \\
\hline No. of parameters & 272 \\
\hline \multirow[t]{2}{*}{$\mathrm{H}$-atom treatment } & $\mathrm{H}$-atom parameters constrained \\
\hline & $w=1 /\left[\sigma^{2}\left(F_{\mathrm{o}}^{2}\right)+(0.0614 P)^{2}+12.4822 P\right]$ \\
\hline
\end{tabular}




\begin{tabular}{|c|c|}
\hline & where $P=\left(F_{\mathrm{o}}^{2}+2 F_{\mathrm{c}}{ }^{2}\right) / 3$ \\
\hline$\left.\Delta\rangle_{\max }, \Delta\right\rangle_{\min }\left(\mathrm{e} \AA^{-3}\right)$ & $2.13,-1.47$ \\
\hline
\end{tabular}

Table S2 Selective bond lengths $[\AA]$ and angles $\left[{ }^{\circ}\right]$ of $\mathbf{1}$.

\begin{tabular}{|c|c|c|c|}
\hline $\mathrm{Au} 1-\mathrm{C} 6$ & $2.027(8)$ & $\mathrm{C} 4-\mathrm{C} 3$ & $1.358(13)$ \\
\hline $\mathrm{Au} 1-\mathrm{C} 7$ & $1.967(10)$ & $\mathrm{C} 3-\mathrm{C} 2$ & $1.360(13)$ \\
\hline $\mathrm{I} 1-\mathrm{C} 19$ & $2.132(11)$ & $\mathrm{C} 13-\mathrm{H} 13$ & 0.9300 \\
\hline $\mathrm{F} 5-\mathrm{C} 5$ & $1.350(10)$ & $\mathrm{C} 13-\mathrm{C} 12$ & $1.374(11)$ \\
\hline $\mathrm{F} 4-\mathrm{C} 4$ & $1.346(10)$ & $\mathrm{C} 9-\mathrm{H} 9$ & 0.9300 \\
\hline $\mathrm{F} 1-\mathrm{C} 1$ & $1.354(10)$ & $\mathrm{C} 12-\mathrm{H} 12$ & 0.9300 \\
\hline $\mathrm{O} 1-\mathrm{C} 11$ & $1.367(9)$ & $\mathrm{C} 12-\mathrm{C} 11$ & $1.394(11)$ \\
\hline $\mathrm{O} 1-\mathrm{C} 14$ & $1.433(11)$ & $\mathrm{C} 2-\mathrm{C} 1$ & $1.389(12)$ \\
\hline $\mathrm{F} 3-\mathrm{C} 3$ & $1.349(9)$ & $\mathrm{C} 18-\mathrm{H} 18 \mathrm{~A}$ & 0.9700 \\
\hline $\mathrm{F} 2-\mathrm{C} 2$ & $1.331(10)$ & $\mathrm{C} 18-\mathrm{H} 18 \mathrm{~B}$ & 0.9700 \\
\hline $\mathrm{O} 3-\mathrm{C} 18$ & $1.396(10)$ & $\mathrm{C} 18-\mathrm{C} 19$ & $1.483(15)$ \\
\hline $\mathrm{O} 3-\mathrm{C} 17$ & $1.414(12)$ & $\mathrm{C} 15-\mathrm{H} 15 \mathrm{~A}$ & 0.9700 \\
\hline $\mathrm{O} 2-\mathrm{C} 15$ & $1.395(12)$ & $\mathrm{C} 15-\mathrm{H} 15 \mathrm{~B}$ & 0.9700 \\
\hline $\mathrm{O} 2-\mathrm{C} 16$ & $1.411(12)$ & $\mathrm{C} 15-\mathrm{C} 14$ & $1.517(13)$ \\
\hline $\mathrm{C} 8-\mathrm{N} 1$ & $1.403(10)$ & C17-H17A & 0.9700 \\
\hline $\mathrm{C} 8-\mathrm{C} 13$ & $1.386(11)$ & C17-H17B & 0.9700 \\
\hline $\mathrm{C} 8-\mathrm{C} 9$ & $1.376(11)$ & $\mathrm{C} 17-\mathrm{C} 16$ & $1.511(14)$ \\
\hline $\mathrm{C} 6-\mathrm{C} 5$ & $1.371(12)$ & $\mathrm{C} 16-\mathrm{H} 16 \mathrm{~A}$ & 0.9700 \\
\hline $\mathrm{C} 6-\mathrm{C} 1$ & $1.383(11)$ & $\mathrm{C} 16-\mathrm{H} 16 \mathrm{~B}$ & 0.9700 \\
\hline $\mathrm{C} 10-\mathrm{H} 10$ & 0.9300 & $\mathrm{C} 19-\mathrm{H} 19 \mathrm{~A}$ & 0.9700 \\
\hline $\mathrm{C} 10-\mathrm{C} 9$ & $1.370(12)$ & С19-H19B & 0.9700 \\
\hline $\mathrm{C} 10-\mathrm{C} 11$ & $1.371(12)$ & $\mathrm{C} 14-\mathrm{H} 14 \mathrm{~A}$ & 0.9700 \\
\hline $\mathrm{N} 1-\mathrm{C} 7$ & $1.143(12)$ & $\mathrm{C} 14-\mathrm{H} 14 \mathrm{~B}$ & 0.9700 \\
\hline $\mathrm{C} 5-\mathrm{C} 4$ & $1.386(11)$ & & \\
\hline $\mathrm{C} 7-\mathrm{Au} 1-\mathrm{C} 6$ & $178.1(3)$ & $\mathrm{O} 3-\mathrm{C} 18-\mathrm{H} 18 \mathrm{~A}$ & 109.7 \\
\hline $\mathrm{C} 11-\mathrm{O} 1-\mathrm{C} 14$ & $119.3(7)$ & $\mathrm{O} 3-\mathrm{C} 18-\mathrm{H} 18 \mathrm{~B}$ & 109.7 \\
\hline $\mathrm{C} 18-\mathrm{O} 3-\mathrm{C} 17$ & $111.9(7)$ & $\mathrm{O} 3-\mathrm{C} 18-\mathrm{C} 19$ & $109.9(8)$ \\
\hline $\mathrm{C} 15-\mathrm{O} 2-\mathrm{C} 16$ & $115.4(8)$ & $\mathrm{H} 18 \mathrm{~A}-\mathrm{C} 18-\mathrm{H} 18 \mathrm{~B}$ & 108.2 \\
\hline
\end{tabular}




\begin{tabular}{|c|c|c|c|}
\hline $\mathrm{C} 13-\mathrm{C} 8-\mathrm{N} 1$ & $118.4(7)$ & $\mathrm{C} 19-\mathrm{C} 18-\mathrm{H} 18 \mathrm{~A}$ & 109.7 \\
\hline $\mathrm{C} 9-\mathrm{C} 8-\mathrm{N} 1$ & $120.2(7)$ & $\mathrm{C} 19-\mathrm{C} 18-\mathrm{H} 18 \mathrm{~B}$ & 109.7 \\
\hline $\mathrm{C} 9-\mathrm{C} 8-\mathrm{C} 13$ & $121.4(7)$ & $\mathrm{O} 1-\mathrm{C} 11-\mathrm{C} 10$ & $125.1(7)$ \\
\hline $\mathrm{C} 5-\mathrm{C} 6-\mathrm{Au} 1$ & $122.9(6)$ & $\mathrm{O} 1-\mathrm{C} 11-\mathrm{C} 12$ & $114.7(7)$ \\
\hline $\mathrm{C} 5-\mathrm{C} 6-\mathrm{C} 1$ & $114.4(7)$ & $\mathrm{C} 10-\mathrm{C} 11-\mathrm{C} 12$ & $120.2(7)$ \\
\hline $\mathrm{C} 1-\mathrm{C} 6-\mathrm{Au} 1$ & $122.7(6)$ & $\mathrm{O} 2-\mathrm{C} 15-\mathrm{H} 15 \mathrm{~A}$ & 110.4 \\
\hline $\mathrm{C} 9-\mathrm{C} 10-\mathrm{H} 10$ & 120.0 & $\mathrm{O} 2-\mathrm{C} 15-\mathrm{H} 15 \mathrm{~B}$ & 110.4 \\
\hline $\mathrm{C} 9-\mathrm{C} 10-\mathrm{C} 11$ & $120.0(7)$ & $\mathrm{O} 2-\mathrm{C} 15-\mathrm{C} 14$ & $106.5(7)$ \\
\hline $\mathrm{C} 11-\mathrm{C} 10-\mathrm{H} 10$ & 120.0 & $\mathrm{H} 15 \mathrm{~A}-\mathrm{C} 15-\mathrm{H} 15 \mathrm{~B}$ & 108.6 \\
\hline $\mathrm{C} 7-\mathrm{N} 1-\mathrm{C} 8$ & $178.0(8)$ & $\mathrm{C} 14-\mathrm{C} 15-\mathrm{H} 15 \mathrm{~A}$ & 110.4 \\
\hline $\mathrm{F} 5-\mathrm{C} 5-\mathrm{C} 6$ & $119.7(7)$ & $\mathrm{C} 14-\mathrm{C} 15-\mathrm{H} 15 \mathrm{~B}$ & 110.4 \\
\hline $\mathrm{F} 5-\mathrm{C} 5-\mathrm{C} 4$ & $116.6(8)$ & $\mathrm{O} 3-\mathrm{C} 17-\mathrm{H} 17 \mathrm{~A}$ & 109.9 \\
\hline $\mathrm{C} 6-\mathrm{C} 5-\mathrm{C} 4$ & $123.7(8)$ & $\mathrm{O} 3-\mathrm{C} 17-\mathrm{H} 17 \mathrm{~B}$ & 109.9 \\
\hline $\mathrm{F} 4-\mathrm{C} 4-\mathrm{C} 5$ & $120.2(8)$ & $\mathrm{O} 3-\mathrm{C} 17-\mathrm{C} 16$ & $108.9(8)$ \\
\hline $\mathrm{F} 4-\mathrm{C} 4-\mathrm{C} 3$ & $120.6(7)$ & $\mathrm{H} 17 \mathrm{~A}-\mathrm{C} 17-\mathrm{H} 17 \mathrm{~B}$ & 108.3 \\
\hline $\mathrm{C} 3-\mathrm{C} 4-\mathrm{C} 5$ & $119.2(8)$ & $\mathrm{C} 16-\mathrm{C} 17-\mathrm{H} 17 \mathrm{~A}$ & 109.9 \\
\hline $\mathrm{F} 3-\mathrm{C} 3-\mathrm{C} 4$ & $119.4(8)$ & $\mathrm{C} 16-\mathrm{C} 17-\mathrm{H} 17 \mathrm{~B}$ & 109.9 \\
\hline $\mathrm{F} 3-\mathrm{C} 3-\mathrm{C} 2$ & $120.4(8)$ & $\mathrm{O} 2-\mathrm{C} 16-\mathrm{C} 17$ & $114.0(8)$ \\
\hline $\mathrm{C} 4-\mathrm{C} 3-\mathrm{C} 2$ & $120.2(7)$ & $\mathrm{O} 2-\mathrm{C} 16-\mathrm{H} 16 \mathrm{~A}$ & 108.8 \\
\hline $\mathrm{C} 8-\mathrm{C} 13-\mathrm{H} 13$ & 120.7 & $\mathrm{O} 2-\mathrm{C} 16-\mathrm{H} 16 \mathrm{~B}$ & 108.8 \\
\hline $\mathrm{C} 12-\mathrm{C} 13-\mathrm{C} 8$ & $118.5(7)$ & $\mathrm{C} 17-\mathrm{C} 16-\mathrm{H} 16 \mathrm{~A}$ & 108.8 \\
\hline $\mathrm{C} 12-\mathrm{C} 13-\mathrm{H} 13$ & 120.7 & $\mathrm{C} 17-\mathrm{C} 16-\mathrm{H} 16 \mathrm{~B}$ & 108.8 \\
\hline $\mathrm{N} 1-\mathrm{C} 7-\mathrm{Au} 1$ & $178.2(8)$ & $\mathrm{H} 16 \mathrm{~A}-\mathrm{C} 16-\mathrm{H} 16 \mathrm{~B}$ & 107.7 \\
\hline $\mathrm{C} 8-\mathrm{C} 9-\mathrm{H} 9$ & 120.2 & $\mathrm{I} 1-\mathrm{C} 19-\mathrm{H} 19 \mathrm{~A}$ & 109.1 \\
\hline $\mathrm{C} 10-\mathrm{C} 9-\mathrm{C} 8$ & $119.6(8)$ & I1- $119-\mathrm{H} 19 \mathrm{~B}$ & 109.1 \\
\hline $\mathrm{C} 10-\mathrm{C} 9-\mathrm{H} 9$ & 120.2 & $\mathrm{C} 18-\mathrm{C} 19-\mathrm{I} 1$ & $112.4(7)$ \\
\hline $\mathrm{C} 13-\mathrm{C} 12-\mathrm{H} 12$ & 119.9 & $\mathrm{C} 18-\mathrm{C} 19-\mathrm{H} 19 \mathrm{~A}$ & 109.1 \\
\hline $\mathrm{C} 13-\mathrm{C} 12-\mathrm{C} 11$ & $120.1(7)$ & $\mathrm{C} 18-\mathrm{C} 19-\mathrm{H} 19 \mathrm{~B}$ & 109.1 \\
\hline $\mathrm{C} 11-\mathrm{C} 12-\mathrm{H} 12$ & 119.9 & $\mathrm{H} 19 \mathrm{~A}-\mathrm{C} 19-\mathrm{H} 19 \mathrm{~B}$ & 107.9 \\
\hline $\mathrm{F} 2-\mathrm{C} 2-\mathrm{C} 3$ & $120.5(8)$ & $\mathrm{O} 1-\mathrm{C} 14-\mathrm{C} 15$ & $107.5(8)$ \\
\hline $\mathrm{F} 2-\mathrm{C} 2-\mathrm{C} 1$ & $120.6(8)$ & $\mathrm{O} 1-\mathrm{C} 14-\mathrm{H} 14 \mathrm{~A}$ & 110.2 \\
\hline $\mathrm{C} 3-\mathrm{C} 2-\mathrm{C} 1$ & $118.9(8)$ & $\mathrm{O} 1-\mathrm{C} 14-\mathrm{H} 14 \mathrm{~B}$ & 110.2 \\
\hline
\end{tabular}




\begin{tabular}{|l|l|c|c|}
\hline $\mathrm{F} 1-\mathrm{C} 1-\mathrm{C} 6$ & $120.0(8)$ & $\mathrm{C} 15-\mathrm{C} 14-\mathrm{H} 14 \mathrm{~A}$ & 110.2 \\
\hline $\mathrm{F} 1-\mathrm{C} 1-\mathrm{C} 2$ & $116.4(7)$ & $\mathrm{C} 15-\mathrm{C} 14-\mathrm{H} 14 \mathrm{~B}$ & 110.2 \\
\hline $\mathrm{C} 6-\mathrm{C} 1-\mathrm{C} 2$ & $123.5(8)$ & $\mathrm{H} 14 \mathrm{~A}-\mathrm{C} 14-\mathrm{H} 14 \mathrm{~B}$ & 108.5 \\
\hline
\end{tabular}

Table S3 Structural comparison with other crystals of similar complexes.

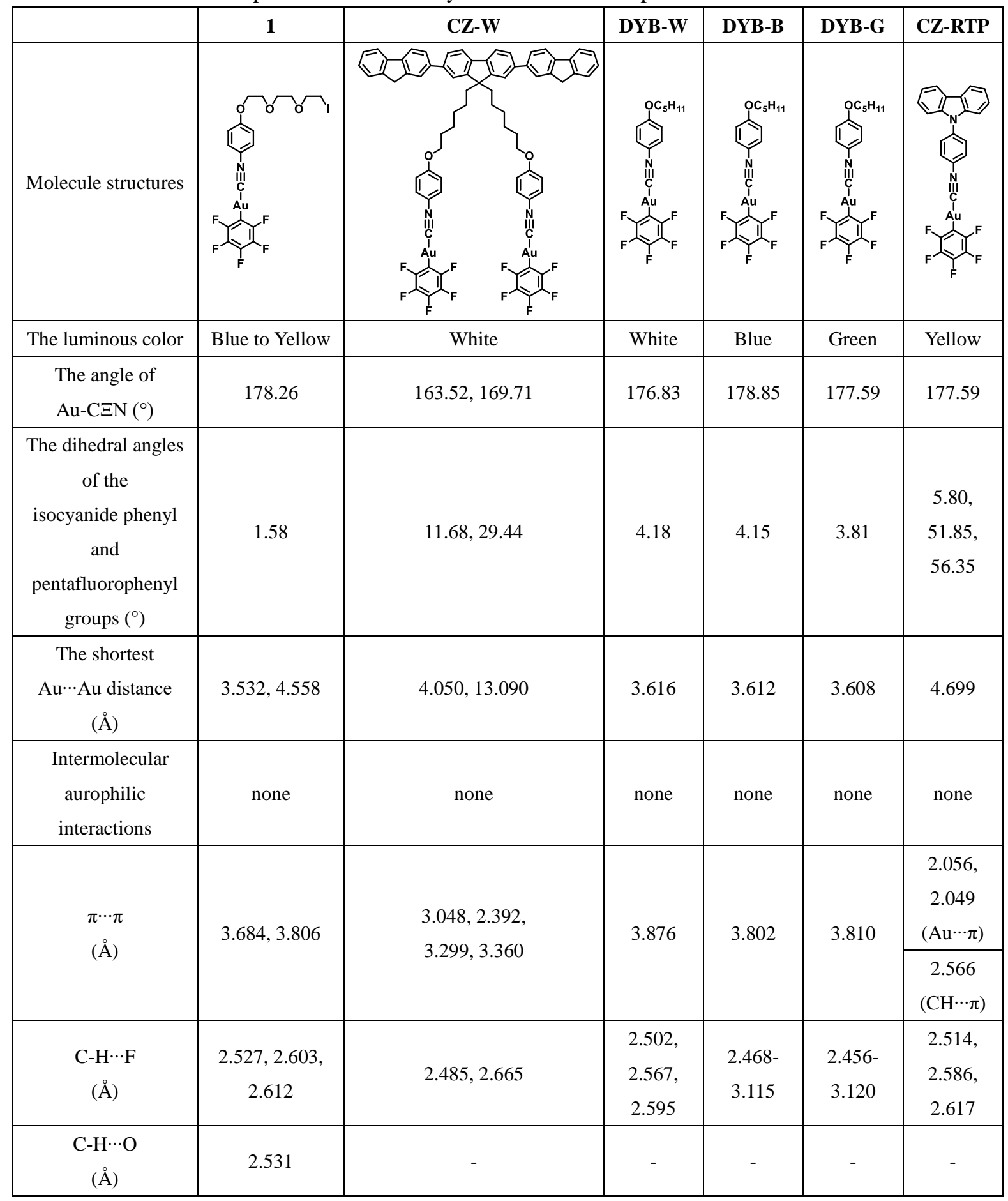

